\title{
Survey of the dependence on temperature of the coercivity of garnet films
}

\author{
G. Vértesy \\ Hungarian Academy of Sciences, Research Institute for Materials Science, H-1525 Budapest, P.O.B. 49, \\ Hungary \\ I. Tomášs \\ Institute of Physics, Academy of Sciences of the Czech Republic, 18040 Praha 8 , Na Slovance 2, \\ Czech Republic
}

(Received 31 May 1994; accepted for publication 16 February 1995)

\begin{abstract}
The temperature dependence of the domain-wall coercive field of epitaxial magnetic garnets films has been investigated in the entire temperature range of the ferrimagnetic phase, and has been found to be described by a set of parametric exponents. In subsequent temperature regions different slopes were observed, with breaking points whose position was found to be sample dependent. A survey based on literature data as well as on a large number of our own samples shows the general existence of this piecewise exponential dependence and the presence of the breaking points. This type of domain-wall coercive field temperature dependence was found in all samples in the large family of the epitaxial garnets (about 30 specimens of more than ten chemical compositions) and also in another strongly anisotropic material (TbFeCo). 1995 American Institute of Physics.
\end{abstract}

\section{INTRODUCTION}

Even in very soft magnetic materials there is always interaction between domain walls and material imperfections. ${ }^{1,2}$ This effect-characterized by the domainwall coercivity-is responsible for the nonzero value of the external magnetic field which is required to move the domain walls irreversibly from their original position. The domainwall coercive field $H_{c w}$ is commonly considered to be one of the most important parameters in applied magnetism connected as it is with the fundamental properties of the material as well as characterizing the degree of perfection of the sample. The domain walls act as probes of the defects, and the value of the measured domain-wall coercive field contains information on the distribution and quality of the defects. Thin magnetic films with nearly perfect crystalline structure are friendly means of investigating subtle details of these ellects. This is particularly true for thin garnet films whose uniaxial magnetic anisotropy has its easy axis normal to the film surface.

The physical properties of magnetic materials determine the nature of the coercivity and the resulting magnetic behavior. To describe the pinning of domain walls at the defects Aharon $i^{3}$ applied the specific Ritz model for the wall structure using a modification of the Hilzinger ${ }^{4}$ theory to study the wall passing a barrier created by planar imperfections; Della Torre ${ }^{5}$ reviewed the problems involved in modeling the coercivity of soft magnetic materials. Many theories (for a review see, e.g., Ref. 1) have also attempted to calculate the coercive field from the material parameters and from an assumed distribution of the material defects. Some of these theories adequately matched the experimental observations. However, the temperature dependence of $H_{c w}$ has been studied only to a limited extent and a considerable decrease of the coercive field with increasing temperature has often been reported. ${ }^{6-11}$ Our results suggest a mathematical expression describing the temperature dependence of $H_{c w}$, and also enable conclusions to be drawn on different types of pinning traps in the material. Recently we investigated the coercive properties of magnetically uniaxial liquid-phase epitaxially grown, rare-earth garnet films from $10 \mathrm{~K}$ up to the Neel temperature $(\approx 480 \mathrm{~K}) .^{12}$ The measured domain-wall coercive field $H_{c w}$ exhibited a steep decrease with increasing temperature $T$. The semilogarithmic plots of $H_{c w}(T)$ revealed the drop to be piecewise exponential, with different slopes at different successive temperature regions. The semilogarithmic temperature dependence of $H_{c w}$ was fitted to the exponential function

$$
H_{c w}=H_{c w o}^{i} \exp \left(-T / T^{i}\right)
$$

in each of its linear parts, where $H_{c w o}^{i}$ and $T^{i}$ were the characteristic values for the $i$ th temperature range. The measured temperature dependence is shown in Fig. 1 for one of the samples used in Ref. 12. In explanation, three different types of wall-pinning traps (material defects) were suggested to coexist in the sample, each of them prevailing in one of the three different temperature regions. The breaking points on the semilogarithmic plot of $H_{c w}(T)$ were identified by the limits of activity of one type of wall-pinning trap and the taking over of the next type. The presence and the position of the three temperaturc ranges of the exponential $H_{c w}(T)$ de crease with the two distinct breaking points in the semilogarithmic plot can be considered to be characteristic of each of the samples.

Our aim here is to find out whether the existence of such temperature ranges, of the breaking points, and of their number and position on the temperature scale are common to many magnetic garnet films (and/or other materials), or if they are characteristic only of some special garnet compositions. With this in mind, $H_{c w}$ versus the temperature data of as many samples with different properties as possible were measured and a survey was carried out of all the available coercivity versus temperature results in the literature.

\section{RESULTS}

General coercive properties of magnetic materials (i.e., their hardness or softness) are usually characterized by the 


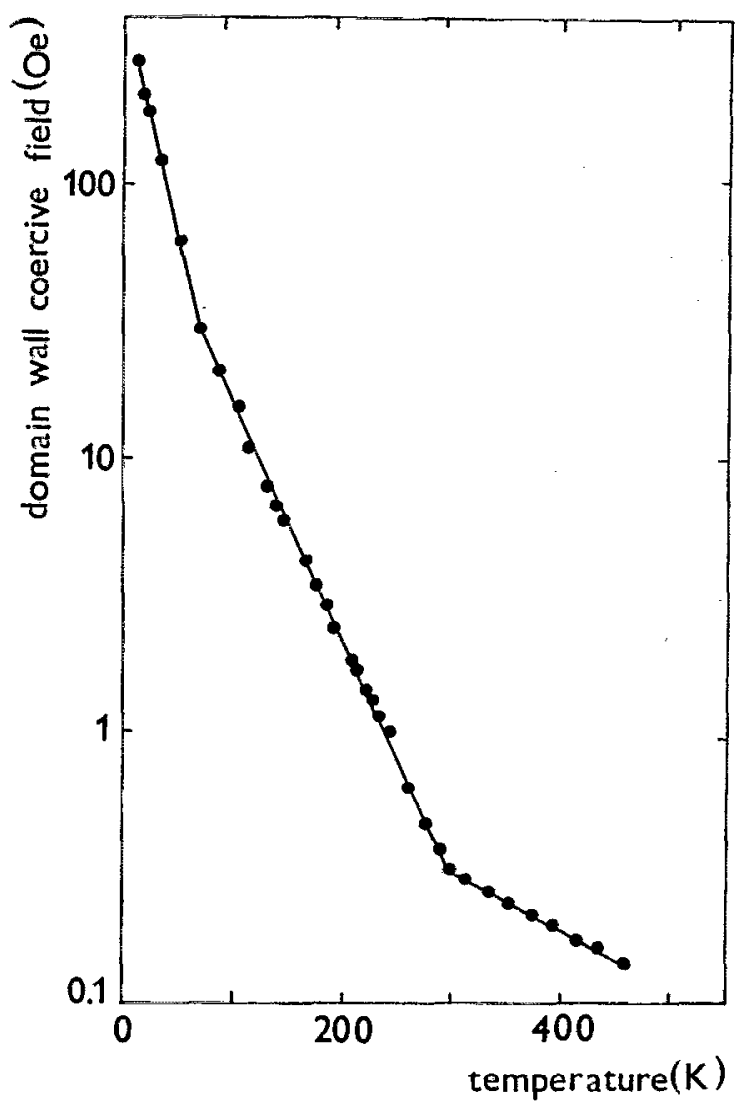

FIG. 1. Temperature dependence of domain-wall coercive field $H_{\mathrm{s} w}$ measured on specimen 6 .

coercive force value $H_{c}$, defined as the half width of the saturation-to-saturation hysteresis loop. However, $H_{c}$ reflects all the complex magnetic changes in the material during its magnetization reversal, including the generation and annihilation of domains, translation and changes of length of domain walls, and rotation of the magnetization vector, but actually there is no straightforward link between the $H_{c}$. value and the behavior of a single domain wall.

If one wishes to address the coercive behavior of the translating domain walls only, finer measuring methods need to be used ${ }^{13}$ and instead of $H_{c}$, the domain-wall coercive field $H_{c w}$ should be determined. For this purpose two methods giving equivalent results of $H_{c w}$, whose error of measurement did not exceed $\pm 8 \%$, were used, viz. lowfrequency domain-wall oscillation, ${ }^{14}$ and the utilization of a vibrating sample magnetometer to measure special minor hysteresis loops. ${ }^{15}$ The former is based on the magnetooptical response of the domain system to an ac magnetic field perpendicular to the film plane. The ac field amplitude is slowly increased from zero and any domain-wall motion is detected photoelectrically. The field amplitude corresponding to the extrapolated start of motion of the domain wall is interpreted as $H_{c w}$.

By contrast, in the latter method the magnetic moment of the sample is measured with the external magnetic field parallel to the easy magnetization axis, i.e., perpendicular to the film plane. The onset of the irreversible domain-wall motion is detected in the measurement.

We measured the temperature dependence of the coercive field of 15 different films with the chemical compositions given in Table I. All the films were grown on [111] oriented GGG substrates by liquid-phase epitaxy. To ensure that specimens with the most different properties were investigated we considered a wide range of coercivity, thickness, saturation magnetization, and quality factor of the chosen films. Some of the specimens even had a compensation point. The parameters of the films were measured in accordance with Ref. 16, and are summarized in Table 1. The measured $H_{c w}(T)$ curves consist of exponential segments for each sample. The number of exponential segments, the temperature range for these segments, and the fitting parameters $T^{i}$ and $H_{c w o}^{i}$ are given for every specimen in Table II. (Not every specimen was measured in the full temperature range

TABLE I. Composition, room-temperature values of domain-wall coercive field $\left(H_{c w}\right)$, film thickness $(h)$, zero-field stripe domain period $\left(p_{0}\right)$, magnetization $\left(4 \pi M_{s}\right)$, uniaxial anisotropy constant $\left(K_{u}\right)$, quality factor $(Q)$, characteristic length $(l)$, and the compensation point $\left(T_{k}\right)$ of the garnet samples which were measured in our laboratory.

\begin{tabular}{|c|c|c|c|c|c|c|c|c|c|}
\hline Specinen & Composition & $\begin{array}{l}H_{C w} \\
(\mathrm{Oe})\end{array}$ & $\begin{array}{c}h \\
(\mu \mathrm{m})\end{array}$ & $\begin{array}{c}p_{0} \\
(\mu \mathrm{m})\end{array}$ & $\begin{array}{c}4 \pi M_{s} \\
(\mathrm{G})\end{array}$ & $\begin{array}{c}\boldsymbol{K}_{u} \\
\left(\mathrm{erg} / \mathrm{cm}^{3}\right)\end{array}$ & $Q$ & $\begin{array}{c}l \\
(\mu \mathrm{m})\end{array}$ & $\begin{array}{l}T_{k} \\
(\mathrm{~K})\end{array}$ \\
\hline 1 & $(\mathrm{YSmCa})_{3}(\mathrm{FeGe})_{5} \mathrm{O}_{12}$ & 0.2 & 5.3 & 9.0 & 211 & 6400 & 4.2 & 0.47 & $\cdots$ \\
\hline 2 & $(\mathrm{YSmCa})_{3}(\mathrm{FeGe})_{5} \mathrm{O}_{12}$ & 0.3 & 4.9 & 9.3 & 203 & 5800 & 3.5 & 0.51 & $\cdots$ \\
\hline 3 & $(\mathrm{YSmCa})_{3}(\mathrm{FeGe})_{5} \mathrm{O}_{12}$ & 1.4 & 5.5 & 17.8 & 147 & 6800 & 7.9 & 1.17 & $\cdots$ \\
\hline 4 & $(\mathrm{YSmCa})_{3}(\mathrm{FeGe})_{5} \mathrm{O}_{12}$ & 2.5 & 5.1 & 10.1 & 195 & 5700 & 3.8 & 0.57 & $\cdots$ \\
\hline 5 & $(\mathrm{YSmCa})_{3}(\mathrm{FeGe})_{5} \mathrm{O}_{12}$ & 3.3 & 5.4 & 10.7 & 198 & 10400 & 6.7 & 0.59 & $\cdots$ \\
\hline 6 & $(\mathrm{YSmCaLu})_{3}(\mathrm{FeGe})_{5} \mathrm{O}_{12}$ & 0.3 & 2.9 & 8.1 & 238 & 15100 & 6.7 & 0.53 & $\cdots$ \\
\hline 7 & $(\mathrm{YSmCaLu})_{3}(\mathrm{FeGe})_{5} \mathrm{O}_{12}$ & 0.9 & 9.2 & 21.5 & 160 & 11000 & 10.8 & 1.30 & $\cdots$ \\
\hline 8 & $(\mathrm{YSmCaLu})_{3}(\mathrm{FeGe})_{5} \mathrm{O}_{12}$ & 1.3 & 2.9 & 7.2 & 305 & 9100 & 2.5 & 0.44 & $\ldots$ \\
\hline 9 & $(\mathrm{YSmCaLu})_{3}(\mathrm{FeGe})_{5} \mathrm{O}_{12}$ & 4.8 & 6.7 & 13.5 & 168 & 5500 & 4.9 & 0.75 & $\ldots$ \\
\hline 10 & $(\mathrm{YSm})_{3}(\mathrm{FeGa})_{5} \mathrm{O}_{12}$ & 0.4 & 4.9 & 9.7 & 191 & 15500 & 10.6 & 0.60 & $\cdots$ \\
\hline 11 & $(\mathrm{YSm})_{3}(\mathrm{FeGa})_{5} \mathrm{O}_{12}$ & 1.8 & 6.7 & 10.5 & 170 & 13000 & 11.3 & 0.51 & $\cdots$ \\
\hline 12 & $(\mathrm{YSm})_{3}(\mathrm{FeGa})_{5} \mathrm{O}_{12}$ & 1.1 & 4.0 & 10.9 & 229 & 16100 & 17.6 & 0.74 & $\cdots$ \\
\hline 13 & $(\mathrm{YYbGdBi})_{3}(\mathrm{FeAl})_{5} \mathrm{O}_{12}$ & 1.7 & 24.5 & 47.0 & 111 & 10800 & 22.0 & 2.60 & 217 \\
\hline 14 & $(\mathrm{EuEr})_{3}(\mathrm{FeGa})_{5} \mathrm{O}_{12}$ & 1.3 & 9.8 & 21.2 & 186 & 25000 & 18.8 & 1.23 & 173 \\
\hline 15 & $(\mathrm{YCa})_{3}(\mathrm{FeCeCo})_{5} \mathrm{O}_{12}$ & 10.8 & 11.8 & 45.0 & 163 & 11700 & 11.0 & 2.98 & 65 \\
\hline
\end{tabular}


TABLE H. Number, temperature range, and fitting parameters of the exponential segments of the garnet samples which were measured in our laboratory. *: not measured in the full temperature range of ferrimagnetic phase, **; above the compensation point, ***: below the compensation point.

\begin{tabular}{|c|c|c|c|c|}
\hline Specimen & $\begin{array}{l}\text { Number of } \\
\text { exponential } \\
\text { segments }\end{array}$ & $\begin{array}{l}\text { Temperature } \\
\text { range } \\
\text { for each } \\
\text { segment } \\
\text { (K) }\end{array}$ & $\begin{array}{l}T^{i}(\mathrm{~K}) \\
\quad \text { for each }\end{array}$ & $\begin{array}{l}H_{x^{w} w}^{i}(\mathrm{Oe}) \\
\text { segment }\end{array}$ \\
\hline & $*$ & $10-95$ & 35.2 & 95 \\
\hline \multirow[t]{3}{*}{1} & 3 & $95-300$ & 57.1 & 23 \\
\hline & & $300-450$ & 129.4 & 0.5 \\
\hline & & $10-70$ & 19.8 & 205 \\
\hline \multirow[t]{2}{*}{2} & 3 & $70-212$ & 61.4 & 18 \\
\hline & & $212-400$ & 113.0 & 3.5 \\
\hline \multirow[t]{2}{*}{3} & $2^{*}$ & $113-265$ & 58.4 & 36 \\
\hline & & $265-453$ & 76.4 & 12 \\
\hline \multirow[t]{3}{*}{4} & $2^{*}$ & $145-274$ & 110.0 & 21 \\
\hline & & $27: 4-345$ & 56.4 & 995 \\
\hline & & $10-144$ & 40.8 & 562 \\
\hline \multirow[t]{3}{*}{5} & 3 & $144-325$ & 107.6 & 51 \\
\hline & & $325-450$ & 229.2 & 10.5 \\
\hline & & $10-90$ & 26.8 & 452 \\
\hline \multirow[t]{2}{*}{6} & 3 & $90-295$ & 47.7 & 105 \\
\hline & & $295-450$ & 97.7 & 1.4 \\
\hline \multirow[t]{3}{*}{7} & $2^{*}$ & $140-223$ & 42.1 & 403 \\
\hline & & $223-440$ & 92.6 & 22 \\
\hline & & $10-58$ & 27.2 & 300 \\
\hline \multirow[t]{3}{*}{8} & 4 & $58-242$ & 118.6 & 51 \\
\hline & & $242-360$ & 32.8 & 9800 \\
\hline & & $340-450$ & 224.2 & 0.8 \\
\hline \multirow[t]{3}{*}{9} & $2^{*}$ & $140-250$ & 63.2 & 36 \\
\hline & & $250-400$ & 193.3 & 480 \\
\hline & & $5-24$ & 108.3 & 652 \\
\hline \multirow[t]{4}{*}{10} & 4 & $24-112$ & 25.5 & 1300 \\
\hline & & $112-248$ & 42.6 & 210 \\
\hline & & $248-380$ & 147.1 & 3.4 \\
\hline & & $10-82$ & 26.9 & 2050 \\
\hline \multirow[t]{2}{*}{11} & 3 & $82-248$ & 44.5 & 680 \\
\hline & & $248-380$ & 135,1 & 15.5 \\
\hline \multirow[t]{4}{*}{12} & $2^{*}$ & $100-242$ & 51.1 & 112 \\
\hline & & $242-380$ & 85.6 & 16.5 \\
\hline & & $225-236$ & 10.5 & $1.8 \times 10^{10}$ \\
\hline & $3^{* * 1}$ & $236-318$ & 76.9 & 81 \\
\hline \multirow[t]{3}{*}{13} & & $318-414$ & 858.0 & 1.8 \\
\hline & $2^{* 3}$ & $150-198$ & -83.3 & 0.5 \\
\hline & & $198-208$ & -6.6 & $2.8 \times 10^{-13}$ \\
\hline \multirow[t]{3}{*}{14} & $1 * *$ & $182-335$ & 34.1 & 1550 \\
\hline & $1 * * *$ & $80-163$ & -24.7 & 0.2 \\
\hline & $2 * *$ & $130-346$ & 51.1 & 2150 \\
\hline \multirow[t]{2}{*}{15} & & $346-412$ & 224.8 & 2.2 \\
\hline & $1 * * *$ & $10-55$ & -45.2 & 140 \\
\hline
\end{tabular}

of the ferrimagnetic order.) A typical $H_{c w}(T)$ curve (measured on specimen 6) is shown in Fig. 1. For the three, well separable exponential segments a least-squares fit to Eq. (1) was performed in every segment; this gave the $T^{i}$ and $H_{c w o}^{i}$ values. The correlation factor of the least-squares fit was $r>0.99$ for each segment.

\section{LITERATURE RESULTS}

Most of the broad enough temperature dependences of the domain-wall coercive field of epitaxial garnets found in the literature were measured by the domain-wall oscillation method. ${ }^{17-22}$ The results were replotted on the semilogarithmic scale. The $H_{c w}(T)$ curves obtained also consist of exponential segments for each sample.

Table III contains the material parameters of every sample taken from the literature. The $T^{i}$ and $H_{c w o}^{i}$ fitting parameters were also calculated for cach exponential segment of every sample, and the results (with the number and temperature range of these segments) are shown in Table IV. The correlation factor of the least-squares fit for the segments was $r \geqslant 0.98$ in every case.

Bubble domain translation ${ }^{23}$ is also frequently used to measure domain-wall coercivity. This method utilizes a highspeed camera to record the velocity of an isolated circular magnetic domain (bubble) in an external magnetic-field gradient, this gradient being produced by a pair of parallel conductors carrying current in the same direction. From the extrapolation of the domain-wall velocity versus drive field curves to zero velocity, the threshold drive field $H_{C w}$ is determined.

The results ${ }^{24-26}$ are also shown in Table IV (samples $\mathrm{N}, \mathrm{O}, \mathrm{P}$ ), and they also exhibit the piecewise exponential decrease of coercivity with increasing temperature.

\section{DISCUSSION}

Since all of the observed cases demonstrate the $H_{c w}=H_{c w o}^{i} \exp \left(-T / T^{i}\right)$ type dependence of the domainwall coercive field, with different slopes of the exponential drop of $H_{c w}$ and no other types of temperature dependence were obtained, our present result supports that of Ref. 12 and indicates that the observed temperature dependence of the domain-wall coercive field is common at least to a large family of garnet films with different chemical composition and grown under different conditions. The difference in the behavior is solely in the number of successive temperature ranges of the constant exponential decrease and the position of the breaking points that can be considered a sample characteristic; these two factors may vary from sample to sample. Possibly some of the samples were measured in too narrow temperature limits to show all ranges of the constant slope, but there seems to be no reason why some samples should not behave in a somewhat different way, i.e., it is possible that certain samples have only two or even only one constant slope of the exponential $H_{c w}(T)$ dependence in the whole ferrimagnetic phase.

The data show that on going from lower to higher temperatures the slope of the $H_{c w}(T)$ plot after the breaking points can be either lower or higher. As suggested in Ref. 12, the direction of the change of the $H_{r w}(T)$ slope is determined by the interaction of the defects and the domain walls. If the decrease of $H_{c w}(T)$ is less rapid at higher temperatures than at lower ones, it is likely that different sets of mutually nonequivalent wall-pinning defects exist in the sample. The defects in different sets are of different quality, strength, and different temperature dependence of their strength. The change of slope of the $H_{c w}(T)$ curve indicates the temperature at which a set of defects takes over the dominant role in the wall coercivity from the previous set of defects. The process is described in detail in Ref. 12. 
TABLE III. Composition, room-temperature values of domain-wall coercive field $\left(H_{c}\right)$, film thickness $(h)$, zeru-field stripe domain period $\left(p_{0}\right)$, magnetization $\left(4 \pi M_{w}\right)$, uniaxial anisotropy field $\left(H_{k}\right)$, quality factor $(Q)$, and characteristic length $(l)$ of the garnet specimens taken from the literature. $(A-M$ : measured by domain-wall oscillation; N-P: measured by bubble translation,

\begin{tabular}{cccccccccc}
\hline \hline Specimen & Composition & $\begin{array}{c}H_{c w} \\
(\mathrm{Oe})\end{array}$ & $\begin{array}{c}h \\
(\mu \mathrm{m})\end{array}$ & $\begin{array}{c}p_{0} \\
(\mu \mathrm{m})\end{array}$ & $4 \pi M_{s}$ & \multicolumn{1}{c}{$\begin{array}{c}H_{k} \\
(\mathrm{Oe})\end{array}$} & $Q$ & $\begin{array}{c}l \\
(\mu \mathrm{m})\end{array}$ & Ref. \\
\hline $\mathrm{A}$ & $(\mathrm{EuCaLu})_{3}(\mathrm{FeGe})_{5} \mathrm{O}_{12}$ & 0.3 & 2.0 & 4.4 & 585 & 2620 & 4.5 & 0.25 & 17 \\
$\mathrm{~B}$ & $(\mathrm{YSmCaLu})_{3}(\mathrm{FeGe})_{5} \mathrm{O}_{12}$ & 0.2 & 2.0 & & 350 & 1630 & 4.3 & 0.27 & 17 \\
$\mathrm{C}$ & $(\mathrm{YSmCa})_{3}(\mathrm{FeGe})_{5} \mathrm{O}_{12}$ & 0.5 & 5.8 & 9.6 & 165 & 460 & 3.8 & 0.50 & 18 \\
$\mathrm{D}$ & $(\mathrm{YSmCa})_{3}(\mathrm{FeGeG})_{5} \mathrm{O}_{12}$ & 1.1 & 6.1 & 16.4 & 98 & 1160 & 12.8 & 1.03 & 18 \\
$\mathrm{E}$ & $(\mathrm{YSmCaLu})_{3}(\mathrm{FeGe})_{5} \mathrm{O}_{12}$ & 0.1 & 5.1 & 9.1 & 235 & 1200 & 5.0 & 0.48 & 19 \\
$\mathrm{~F}$ & $(\mathrm{YSmCaLu})_{3}(\mathrm{FeGe})_{5} \mathrm{O}_{12}$ & 0.2 & & & & 1400 & 6.0 & & 19 \\
$\mathrm{G}$ & $(\mathrm{YSmCaLu})_{3}(\mathrm{FeGe})_{5} \mathrm{O}_{12}$ & 0.3 & 1.8 & & 490 & 723 & 1.5 & 0.16 & 20 \\
$\mathrm{H}$ & $(\mathrm{YSmCaLu})_{3}(\mathrm{FeGe})_{5} \mathrm{O}_{12}$ & 0.3 & 2.2 & & 359 & 1259 & 3.5 & 0.29 & 20 \\
$\mathrm{I}$ & $(\mathrm{YSmCaLu})_{3}(\mathrm{FeGe})_{5} \mathrm{O}_{12}$ & 0.5 & 2.5 & & 419 & 1968 & 4.7 & 0.26 & 20 \\
$\mathrm{~J}$ & $(\mathrm{EuEr})_{3}(\mathrm{FeAlGa})_{5} \mathrm{O}_{12}$ & 0.9 & & & 166 & 2270 & & 0.76 & 21 \\
$\mathrm{~K}$ & $(\mathrm{EuEr})_{3}(\mathrm{FeGa})_{5} \mathrm{O}_{12}$ & 1.4 & & & 227 & 4620 & & 0.91 & 21 \\
$\mathrm{~L}$ & $(\mathrm{YEuYb})_{3}(\mathrm{FeAl})_{5} \mathrm{O}_{12}$ & 0.3 & & & 239 & 1230 & & 0.38 & 21 \\
$\mathrm{M}$ & $(\mathrm{TmBi})_{3}(\mathrm{FeGa})_{5} \mathrm{O}_{12}$ & 0.8 & 5.0 & 9.8 & 200 & & & 0.55 & 22 \\
$\mathrm{~N}$ & $(\mathrm{YBiCa})_{3}(\mathrm{FeSi})_{5} \mathrm{O}_{12}$ & 0.2 & 0.9 & & & 1000 & & & 24 \\
$\mathrm{O}$ & $(\mathrm{YSmCaLu})_{3}(\mathrm{FeGe})_{5} \mathrm{O}_{12}$ & 0.3 & 5.1 & & 227 & & 6.0 & 0.55 & 25 \\
$\mathrm{P}$ & $(\mathrm{YSm})_{3}(\mathrm{FeGa})_{5} \mathrm{O}_{12}$ & 0.7 & 3.9 & 7.5 & 275 & 1500 & & 0.42 & 26 \\
\hline \hline
\end{tabular}

TABLE IV. Number, temperature range, and fitting parameters of the exponential segments of the garnet specimens taken from the literature. (The specinnens were not measured in the full temperature range of ferrimagnetic phase.)

\begin{tabular}{|c|c|c|c|c|}
\hline Spatinen & $\begin{array}{l}\text { Number of } \\
\text { exponential } \\
\text { segments }\end{array}$ & $\begin{array}{l}\text { Temperature range } \\
\text { for aach segment } \\
\text { (K) }\end{array}$ & $\begin{array}{l}T^{3}(\mathrm{~K}) \\
\quad \text { for each }\end{array}$ & $\underset{\text { segment }}{H_{e w n}^{i}}(\mathrm{Oe})$ \\
\hline \multirow[t]{2}{*}{$A$} & 2 & $230-261$ & 44.3 & 142 \\
\hline & & $261-370$ & 76.7 & 12 \\
\hline \multirow[t]{2}{*}{$B$} & 2 & $240-294$ & 51.0 & 102 \\
\hline & & $294-360$ & 51.0 & 102 \\
\hline \multirow[t]{2}{*}{$\mathrm{C}$} & 2 & $80-128$ & 47.4 & 78 \\
\hline & & $12 x-310$ & 73.1 & 30 \\
\hline \multirow[t]{2}{*}{ D } & 2 & $80-141$ & 54.5 & 130 \\
\hline & & $141-320$ & 74.6 & 65 \\
\hline \multirow[t]{2}{*}{$\mathrm{E}$} & 2 & $230-315$ & 43.0 & 136 \\
\hline & & $315-360$ & 22.7 & 93900 \\
\hline \multirow[t]{2}{*}{$\mathrm{F}$} & 2 & $230-313$ & 42.5 & 251 \\
\hline & & $313-380$ & 61.2 & 27 \\
\hline \multirow[t]{2}{*}{$\mathrm{G}$} & 2 & $220-309$ & 83.5 & 13 \\
\hline & & $309-380$ & 312 & 6171 \\
\hline \multirow[t]{2}{*}{$\mathrm{H}$} & 2 & $220-277$ & 49.1 & 105 \\
\hline & & $277-380$ & 105.5 & 5.1 \\
\hline \multirow[t]{2}{*}{ I } & 2 & $220-316$ & 103.3 & 10 \\
\hline & & $316-380$ & 44.5 & 565 \\
\hline \multirow[t]{2}{*}{$\mathrm{J}$} & 2 & $250-333$ & 80.8 & 33 \\
\hline & & $333-355$ & 666.7 & 0.9 \\
\hline \multirow[t]{2}{*}{$\mathrm{K}$} & 2 & $250-353$ & 58.8 & 109 \\
\hline & & $353-375$ & 17.2 & $2.1 \times 10^{8}$ \\
\hline \multirow[t]{2}{*}{$\mathcal{L}$} & 2 & $160-272$ & 51.6 & 91 \\
\hline & & $272-360$ & 32.3 & 2140 \\
\hline \multirow[t]{2}{*}{$\mathrm{M}$} & 2 & $160-276$ & 60.4 & 7.7 \\
\hline & & $276-410$ & 262,8 & 2.3 \\
\hline \multirow[t]{2}{*}{$\mathrm{N}$} & 2 & $210-333$ & 118.8 & 2.8 \\
\hline & & $333-420$ & 625.0 & 0.3 \\
\hline \multirow[t]{2}{*}{0} & 2 & $230-298$ & 84.4 & 10 \\
\hline & & $298-350$ & 41.3 & 408 \\
\hline \multirow[t]{2}{*}{$\mathrm{P}$} & 2 & $230-295$ & 87.1 & 24 \\
\hline & & $295-340$ & 307.7 & 2.1 \\
\hline
\end{tabular}

If the decrease of $H_{c w}(T)$ is more rapid at higher temperatures, overlapping of the potential wells of individual defects of the same set of pinning traps probably occurs. The break point marks the interaction of the pinning traps when-due to the increased temperature-the potential wells of the defects become broader and their slopes start to overlap. This overlapping causes a more rapid decrease of their slopes than before they started to merge. No case was found of two breaking points following cach other with increasing slope of the $H_{c w}(T)$ curve with increasing temperature.

In that we measured both directions of the change of the slopes, it seems probable that both kinds of pinning interaction models exist in epitaxial garnet films.

From the above arguments, it would appear that different sets of material imperfections are responsible for the pinning of the domain walls. If this is indeed the case, it can be expected that by appropriate processing some of the pinning sets can be annihilated, generated, or at least modified which, in turn, could modify of the temperature dependence of the domain-wall coercive field. Systematic investigation of a large series of processed garnet thin-film samples is presently under way in our laboratory. The results-hinting as to the origin of the wall-pinning defects-will be submitted for publication in the near future.

The described temperature dependence of $H_{r^{\prime}}$ reflects the presence, the temperature behavior, and the mutual interaction of the wall-pinning traps rather than details of the walls and their behavior. As mentioned above various methods can be found for measuring the coercivity. Any direct comparison of the coercivity values, measured in different laboratories by different techniques, is not simple because different samples are used for the measurements in view of which in a previous paper ${ }^{13}$ the coercivity was measured on the same sample by many different methods. The domainwall oscillation methods were found to characterize the domain-wall-defect interactions in the most appropriate way and actually almost no correlation was found betwcen the 
room-temperature results of the wall oscillation method and those from bubble translation. In spite of this, similar temperature dependence exists even for the coercivities obtained by these two different methods. They both show the general behavior of the exponential dependence of $H_{c w}(T)$, and also the existence of the breaking points.

Due to the high uniaxial anisotropy the domain structure of garnet films is usually simple; the direction of magnetization inside the neighboring domains is antiparallel and normal to the films' surface. The domain walls are $180^{\circ}$ Bloch walls in the center and approach Néel walls at the surface. As the walls meander, they bow out therefore their thickness may vary throughout their depth. ${ }^{27}$ The structure of the walls depends on the character of the material and also on the growth conditions. However, the observed and reported temperature dependence of coercivity is believed not to be specific to the detailed structure of the walls. Measurements carried out on ion implanted garnet films support this assumption.

The domain walls are not usually simple, divergencefree Bloch walls even in the center, but contain alternating Bloch- and Néel-type segments. Néel segments behave like a wall between the Bloch segments, and are known as Bloch lines. The presence of these lines distinguishes a so-called hard wall from a normal one, and there is an increase of the domain-wall energy in hard walls due mainly to the exchange energy of the Bloch lines-as was shown in Refs. 28 and 29. The increased energy of hard walls gives rise to increased coercivity. Hard walls can be suppressed by ion implantation, ${ }^{30,31}$ which creates an approximately $0.1 \mu \mathrm{m}$ thick surface layer with a large number of defects and with modified anisotropy necessary to eliminate Bloch lines. The anisotropy change is the highest at a dose of $2 \times 10^{14} \mathrm{Ne}^{+}$ ions $/ \mathrm{cm}^{2}$ at $80-100 \mathrm{keV}$ energy (for a strain of $1.1 \%$ ); at higher doses and strains the implanted layer becomes amorphous.

Specimen 1 (Table I) was cut into several pieces of which two were implanted: one piece was implanted by 80 $\mathrm{keV} \mathrm{Ne}{ }^{+}$ions with a dose of $2 \times 10^{14}$ ions $/ \mathrm{cm}^{2}$ to achieve the highest anisotropy change; another piece of the same specimen was implanted by $110 \mathrm{keV}$ Xe ions with a dose of $1 \times 10^{15}$ ions $/ \mathrm{cm}^{2}$ to produce as much damage in the surface layer as possible. The temperature dependence of the domain-wall coercive field of these pieces was remeasured after the ion implantation. No change was observed in the $H_{c w}(T)$ curve; the same exponential segments were measured for a nonimplanted specimen. In spite of the fact that the fine structure of the domain walls was substantionally modified, no measurable change was observed in the temperature dependence of coercivity.

\section{CONCL.USIONS}

The temperature dependence of the domain-wall coercive field of about 30 specimens with more than ten different chemical compositions of epitaxially grown magnetic garnets is shown and discussed in the present paper. All of the specimens both from our laboratory and from the literature behaved in a similar way, i.e., their domain-wall coercive field dropped piecewise exponentially with increasing tem- perature. Up until now, no rare-earth epitaxial garnet has been found to behave differently. This observation leads us to suggest that this $H_{c w}(T)$ dependence is typical for this group of materials.

As the explanation of the shape of the $H_{c w}(T)$ curves (see Ref. 12) is connected with a general way of mutual interaction of the wall-piming traps rather than with their concrete origin, similar $H_{C w}(T)$ behavior can be expected in other materials whose coercivity (the strength of the wallpinning traps) is a steep function of temperature.

A detailed survey of materials other than garnets has not yet been done, but the above assumption is supported by the measurements of Perlov et al. ${ }^{32}$ and Della Torre et al. ${ }^{33}$ who measured the temperature dependence of coercivity of $\mathrm{Tb}$ $\mathrm{FeCo}$ films used in magneto-optic recording. Their results seem to be very similar to those obtained on gamets.

\section{ACKNOWLEDGMENTS}

The authors are indebted to Dr. B. Keszei and Dr. J. Vandlik for the growth of the samples. The financial support (Grant No. 19058) from the Grant Agency of the Academy of Sciences of the Czech Republic and from the PECO Project (Contract No. CIPA-CT93-0239) is appreciated.

${ }^{1}$ M. Pardavi-Horvăth, IEEE Trans. Magn. MAG-21, 1694 (1985).

${ }^{2}$ A. N. Grigorenko, S. A. Mishin, and E. G. Rudasherskii, Solid State Phys. (USSR) 30, 2948 (1988).

${ }^{3}$ A. Aharoni, J. Appl. Phys. 58, 2677 (1985).

${ }^{4}$ H. R. Hilzinger, Appl. Phys. 12, 253 (1977).

${ }^{5}$ E. Della Torre, J. Phys. Paris 49, C8-1909 (1988).

${ }^{6}$ D. W. Camegie and H. Claus, Phys. Rev. B 20, 1280 (1979).

${ }^{7}$ D. A. Read, T. Moyo, and G. C. Hallam, J. Magn. Magn. Mater. 44, 279 (1984); 54-57, 309 (1986).

${ }^{8}$ D. G. Rancourt, S. Chehab, and G. Lamarche, J, Magn. Magn. Mater, 78, 129 (1989).

${ }^{9}$ P. Gaunt, Philos. Mag. B 48, 261 (1983).

${ }^{10} \mathrm{~J}$. Maguin, H. Jouve, and B. Barbara, J. Appl. Phys. 50, 1538 (1979),

${ }^{11} \mathrm{H}$. Komatsu. K. Fukamichi, N. Sato, and Y. Nakagawa, IEEE Trans. J. Magn. Jpn. 4, 270 (1989).

${ }^{12}$ G. Vertesy. I. Tomás, L. Pŭst, and J. Paces, J. Appl. Phys. 71, 3462 (1992).

${ }^{13}$ G. Vértesy, M. Pardavi-Horváth, L. Bódis, and I. Pintér, J. Magn. Magn. Mater, 75, 389 (1988).

${ }^{14}$ J. A. Seitchik, G. K. Goldberg, and W. D. Doyle, J. Appl. Phys, 42, 127 ? (1971).

${ }^{15}$ G. Vértesy, L. Pưst, and I. Tomíš, J. Phys. D 24, 1482 (1991).

${ }^{16}$ R. M. Josephs, Am. Inst. Phys. Conf. Proc. 10, 286 (1972).

${ }^{17}$ B. Ferrand, H. Moriceau, D. Challeton, and J. Daval, IEEE Trans. Magn. MAG-14, 415 (1978).

${ }^{18}$ S. Bornmann, P. Görnert, V. A. Bokov, M. V. Bistrov, and V. A. Yatsenko, Solid State Phys. (USSR) 21, 3687 (1979).

${ }^{19}$ G. G. Summer and W. R. Cox, Am. Inst. Phys. Conf. Proc. 34, $157(1976)$.

${ }^{20}$ W. R. Cox and S. G. Parker, Mater. Res. Bull. 13, 501 (1978).

${ }^{21}$ P. W. Shumate, Jr. D. H. Smith, and F. B. Hagedorn, J. Appl. Phys. 44, 449 (1973).

${ }^{22}$ V. A. Zablotskii, Yu. A. Mamalui, and Yu. A. Siryuk, J. Phys. Condens. Matter 4, 2623 (1992).

${ }^{23}$ G. P. Vella-Coletro and W. J. Tabor, Appl. Phys. Lett. 21, 7 (1972).

${ }^{24}$ S. E. G. Slusky, J. E. Ballantine, R. A. Lieberman, L. C. Luther, and T. J. Nelšon, IEEF Trans, Magn. MAG-18, 1286 (1982).

${ }^{25}$ R. E. Fontana, Jr. and D. C. Bullock, Am. Inst. Phys. Conf. Proc. 34, 170 (1973).

${ }^{26}$ P. Pougnet, H. Jouve, and B. Barbara, J. Magn. Magn. Mater. 27, 109 (1982).

${ }^{27}$ A. P. Malozemoff and J. C. Slonczewski, Magnetic Domain Walls in 
Bubble Muterials (Academic, New York, 1979).

${ }^{28}$ A. Rosencwaig, W. J. Tabor, and T. J. Nelson, Phys. Rev. Lett. 29, 946 (1972).

A. A. Thiele, J. Appl. Phys. 45, 377 (1974)

${ }^{3} \mathrm{R}$. Wolfe and J. C. North, Bell Syst. Tech. J. 51, 1436 (1972).
${ }^{31}$ H. L. Lu and E. A. Giess, IEEE Trans. Magn. MAG-11, 1085 (1975).

${ }^{32}$ C. M. Perlov, E. Della Torre, and H. Birecki, J. Appl. Phys. 67, 4444 (1990).

${ }^{33}$ E. Della Torre, C. M. Perloy, and M. Pardavi-Horvath, J, Magn. Magn. Mater. 104-107, 303 (1992). 